\title{
A NEW ALGORITHM FOR GAUSS MARKOV IDENTIFICATION
}

\section{P. BERNHARD $(*)$}

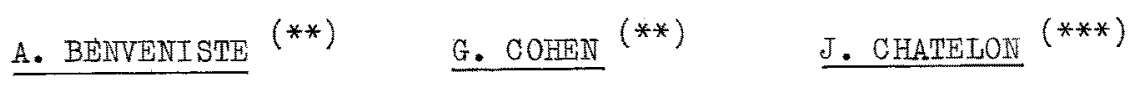

\section{SUIMIARY.}

Based on the theory of Gauss Markov representation established by FAURRE [4], and on a new theorem about the inverse of the spectrum of the signal process, a new algorithm is proposed, using frequency methods, but which does not appeal to classical spectral factorization, nor to any algebraic Riccati equation.

\footnotetext{
(*) Centre d'Automatique de I'Ecole Nationale Supérieur e des Mines de Paris, Fontainebleau, and IRIA, Rocquencourt, France.

(**) Centre d'Automatique de I'Ecole Nationale supérieue des Mines de Paris, Fontainebleau, France.

(***) Centre d'Automatique de I'Ecole Nationale Supérieue des Mines de Paris, Fontainebleau, France, and ECAM France.
} 


\section{Introduction.}

The Kalman filtering theory starts from the following internal description of the signal $y($.$) to be filtered :$

$$
\begin{aligned}
& x(k+1)=F x(k)+v(k), \\
& y(k)=H x(k)+w(k) .
\end{aligned}
$$

Here, $x(k) \in R^{n}, y(k) \in R^{m},\left(v^{\prime}, w^{\prime}\right)^{(1)}$ is a white noise of appropriate dimension, characterized by

$$
\mathrm{E}\left(\begin{array}{l}
\mathrm{v}(\mathrm{k}) \\
w(\mathrm{k})
\end{array}\right)=\left(\begin{array}{l}
0 \\
0
\end{array}\right), \mathrm{E}\left(\begin{array}{c}
\mathrm{v}(\mathrm{k}) \\
w(\mathrm{k})
\end{array}\right) \quad\left(\mathrm{v}^{\prime}(\mathrm{k}) \mathrm{w}^{\prime}(\mathrm{I})\right)=\left(\begin{array}{ll}
\mathrm{Q} & \mathrm{S} \\
\mathrm{S}^{\prime} & \mathrm{R}
\end{array}\right) \delta_{\mathrm{KI}} \cdot
$$

F, H, Q, $S$ and $R$ are constant matrices, $F$ is asymptotically stable, $Q=I^{\prime}$ with (F, I) completely controllable, and $R$ is assumed positive definite (regular case).

on the other hand, Wiener filtering theory starts from the following external description of the process :

$$
\Lambda_{\mathrm{k}}=\mathrm{E}\left[\mathrm{y}(\mathrm{k}+1) \mathrm{y}^{\prime}(1)\right]
$$

Whe $\Lambda_{k}$ can be directly measured from a realization of $y(\cdot)$ provided the signal process is ergodic.

The aim of Gauss Markov identification is to fill the gap between the two approaches. More precisely, the object is to be able to construct the Kalman filter of a process knowing only its external description. One approach whould be to use classical wiener filtering techniques, and then find an internal description of the filter obtained. The obstacle there is the spectral factorization which turns out to be difficult to perform for a matrix spectrum.

The alternate approach is to find an internal representation of the process, and then compute the Kalman filter via classical mears. The drawback of this method was that two algebraic matrix Riccati equations

(1) a prime on a vector or matrix means "transposed". 
had to be solved : one to find the internal description, one to compute the filter gain. FAURRE [4] has shown that this number can be reduced to one by finding precisely the filter as one particular internal representation of $\mathrm{y}$. As a matter of fact, it was pointed out by MEHRA [7] that the filter can be written as

$$
\begin{aligned}
& \hat{\mathrm{x}}(\mathrm{k}+1)=\mathrm{F} \hat{\mathrm{x}}(\mathrm{k})+\operatorname{Tn}(\mathrm{k}) \\
& \mathrm{y}(\mathrm{k})=\mathrm{H} \hat{\mathrm{x}}(\mathrm{k})+\eta(\mathrm{k})
\end{aligned}
$$

and it is know that the innovation process $\eta(k)$ is white. What is often sought is the Wiener estimate $\hat{y}=H \hat{x}$.

In this paper, we give a new way of finding the filter from the $\Lambda_{\mathrm{k}}{ }^{\prime} \mathrm{s}$, or more precisely from the spectrum of $\mathrm{y}$ :

$$
\Gamma(z)=\sum_{k=-\infty}^{+\infty} \Lambda_{k} z^{-k}
$$

We carry out all the analysis in discrete time as it is the formulation which is computationally usefull, but it carries over to the continuous case (it is only simpler). Let us point out also that by duality it applies to the linear quadratic stable regulator problem. See $[1],[8]$.

\section{Spectrum of $y($.$) .$}

It is well known that the link between (1) (2) on one hand and (3) on the other, is given, using

$$
P=E\left[x(k) x^{y}(k)\right],
$$

which is computed through (9), and

$$
\mathrm{G}=\mathrm{E}\left[\mathrm{x}(\mathrm{k}+1) \mathrm{y}^{\prime}(\mathrm{k})\right] \text {, }
$$

which is usually defined by $(10)$, by the equations :

$$
\begin{aligned}
& P-F P F^{2}=Q, \\
& G-F_{P H}^{\prime}=S, \\
& \Lambda_{O}-H P H^{\prime}=R, \\
& \Lambda_{k}-H H^{k-1} G=0, k \geq 1 .
\end{aligned}
$$


$\Lambda_{\mathrm{k}}$ depends only on $\mathrm{H}, \mathrm{F}$ and $\mathrm{G}$. Therefore, for fixed $\Lambda_{0}, \mathrm{H}, \mathrm{F}, \mathrm{G}$, placing various non negative definite $\mathrm{P}^{\prime} \mathrm{s}$ in $(9),(10),(11)$, we obtain various triples ( $Q, S, R$ ) that all give the same external description. All the se models will be termed "equivalent".

such a model has a meaning only if $P$ is such that the resulting noise covariance matrix built with ( $Q, S, R$ ) is non negative definite. However, one can show that the filter can be computed from any of these models (meaningfull or not), and that all have the same filter. (i.e. the same gain $\mathrm{T}$ in (4)).

From (12), it is clear that the spectrum $\Gamma(z)$ of $y$ can be written

$$
\Gamma(z)=\Lambda_{0}+H(z I-F)^{-1}\left(G+G^{\prime}\left(\frac{1}{z} I-F^{\prime}\right)^{-1} H^{\prime}\right.
$$

DEFINITION. A triple ( $H, F, G)$ satisfying (13) is called an additive realization of $\Gamma$. It is said minimal if the dimension of $F$ is minimal among all such realizations.

F being, by hypothesis, asymptotically stable, the additive decomposition of $\Gamma$ is unique, with the first term constant, the second realizable and the third antirealizable. Then, it follows from classical realization theory [6] that all minimal realizations are identical up to a change of basis in the state space.

The result on which the new algorithm is based is the following :

THEORHM. There exists a minimal additive realization $(\bar{H}, \bar{F}, \bar{G})$ of $\overline{\Gamma^{i}-1}(z)$ with

$$
\begin{aligned}
& \overline{\mathrm{H}}=\mathrm{T}^{\prime} \\
& \overline{\mathrm{F}}=(\mathrm{F}-\mathrm{TH})^{\prime}
\end{aligned}
$$

COROIIARY. The matrix $F-T H$ is asymptotically stable iff the inverse of the spectrum has no pole on the unit circle.

For a proof of the theorem, see [2] which gives a more complete account of the present theory and [1]. The corollary follows immediately.

\section{Algorithms}

All algorithms first involve evaluation of the $\Lambda_{k}{ }^{\prime}$ s. An efficient 
way of doing so is via the spectrum $\Gamma\left(e^{i T \omega}\right)$ for discrete values of $\omega$. (And using fast FOURIER transforms : FFT's). Then, from the $\Lambda_{k}{ }^{\prime} s$, a standard realization algorithm yields $H, F$ and $G$ (see [5], [3].

The algorithm we propose is to invert $\Gamma\left(e^{i T \omega}\right)$ for all values of $\omega$. Then, do a FFT, obtain a sequence $\nabla_{k}$, and a realization algorithm as previously. Obtain matrices $H$ and $F$. If a filter for $x$ were sought this would suffice :

$$
\hat{x}(k+1)=\bar{F}^{\prime} x(k)+\bar{H}^{\prime} y(k)
$$

However, $x$ has no meaning of its own, and is defined up to a change of basis. Thus we want $\hat{y}=$ Fix. But if we have realized both the sequence $\Lambda_{\mathrm{k}}$ and $V_{\mathrm{k}}$, the matrices obtained are usually not in the same basis. We must therefore match these bases. Having obtained the two realizations, there exists a non singular matrix $\mathbb{M}$ such that

$$
\begin{aligned}
& \overrightarrow{\mathrm{F}}^{\prime}=\mathrm{M}(\mathrm{F}-\mathrm{TH}) \mathrm{M}^{-1} \\
& \overrightarrow{\mathrm{H}}^{-1}=\mathrm{MP}
\end{aligned}
$$

Hence knowing $\mathrm{H}, \mathrm{F}, \overline{\mathrm{H}}$ and $\overline{\mathrm{F}}$, we can compute $\mathrm{M}$ :

$$
F^{\prime} M-M H^{\prime}=-\vec{H}^{\prime} H
$$

This is a linear equation for $\mathbb{M}$. Then $T$ can be obtained from (15). Germain, of IRIA, pointed out to us that when $y$ is scalar, we can dispense with this equation by realizing both the $V_{\underline{k}}{ }^{\prime}{ }^{\prime}$ and the $\Lambda_{k}{ }^{\prime} s$ in companion form (which Rissanen's algorithm does). Then, the coefficient of $T$ is just the difference of those in $F$ and $\bar{F}^{*}$. (It might be possible to do the same thing for the multivariable case, using an appropriate canonical form).

\section{Conclusion.}

The main contribution of this paper, as we see it, is to show that the algebraic Riccati equation, or a substitute, is by no means a necessary tool apart from standard spectral factorization.

Now, it is interesting to discuss the relative merits of this algorithm as compared to FAURRE"s [4], which is discussed in parallel in $[2]$. 
Its drawbacks are the need for three FFT's instead of two, and two realization algorithms instead of one. However these are extremely performing algorithms, and it is not a very serious problem. Another drawback is the necessity to match the bases. We have seen that this could be improved upon.

The good point is replacing the Riccati equation by a sequence of matrix inversions. Notice that the Riccati equation involves a matrix inversion of the same dimension at each step. But this is nothing as compared to the real numerical dificulties associated with this equation, as is well known in the literature. And it is of dimension $n$, larger that the dimension $m$ of $\Gamma$.

Therefore, one should expect that the new algorithm is more efficient every time the.dimension of the state vector is large as compared to that of the signal.

\section{BIBLIOGRAPHY.}

[1] P. BERNHARD et $G$. COHEN : "Etude d une fonction frequentielle intervenant en commande optimale avec une application à la réduction de la taille de ce problème". R.A.I.R.0, yellow series, J-2., 1973.

[2] P. BERNHARD et al. "Same title as this paper". Internal report A/54 of Centre d'Automatique de 1'ENSMP, Fontainebleau, France 1974.

[3] P. FAURRE et CHATAIGNER : "Identification en temps réel et en temps différé par factorisation de matrices de Hankel" proceeding, FrenchSwedish symposium on process control, IRIA, 1971.

[4] P. FAURRE : "Representation markovienne de processus aléatoires". IRIA report n० 13, IRIA, France, 1973.

[5] B. I. HO, RE. KAIMAN : "Effective construction of linear state variable models from input/output functions". Proc. 3rd Allerton Conference, pp 449-459, 1965 .

[6] R.E. KATMAN. "Modern theory of realization" in. Kalman, FAIB and ARBIB : Topics in Mathematical System Theory (Chapt 10). McGraw Hill, 1969 .

[7] MEHRA : "On the Identification of variances and adaptive Kalman filtering". IEEE transactions on A.C., AC-15, $\mathrm{n}^{\circ} 2$, pp 175-184, 1970.

[8] J.C. WILUEMS. : "least squares stationary optimal control and the algebraic Riccati equation", IEEE transactions on A.C., $\mathrm{AC}-16$, pp 621-634, 1971 . 